\title{
Hyperpigmentation with Vitamin B12 Deficiency in an Adolescent Girl
}

\author{
SH HASAN ${ }^{\mathrm{a}}, \mathrm{R} \mathrm{SAMAD}^{\mathrm{b}}, \mathrm{JC}^{\mathrm{DAS}}{ }^{\mathrm{c}}, \mathrm{F} \mathrm{ARA}^{\mathrm{d}}$, MA FAISAL $^{\mathrm{e}}$
}

\begin{abstract}
:
Hyperpigmentation as primary symptom is rare in vitamin B12 deficiency. This adolescent non vegetarian girl presented with hyperpigmentation, anaemia, glossitis and weight loss. She was worked up to find out the cause of anemia and hyperpigmentation. Addison disease was a strong provisional diagnosis. Her cortisol level was normal and megaloblastic anemia due to vitamin $B 12$ deficiency was found. All of her symptoms were
\end{abstract}

Introduction:

Vitamin B12 plays a major role in human intermediary metabolism. ${ }^{1}$ The main dietary sources of B12 are animal products including meat, fish, eggs, and dairy products. B12-containing fortified plant products may be the other source. ${ }^{2}$ Deficiency of Vitamin B12 can affect all age groups but the elderly and the vegetarians/lactovegetarian are at highest risk. ${ }^{2,3}$ Signs and symptoms arising out of vitamin B12 deficiency are varied and non-specific. The main systems affected are the hematologic, gastrointestinal, and nervous system. ${ }^{3}$ Some of the lesser known manifestations are cutaneous such as

a. Dr. Syeda Humaida Hasan, MBBS, DCH, FCPS (Pediatrics), Consultant, Department of Neonatology, Chittagong Medical College Hospital, Chittagong, Bangladesh.

b. Dr. Rasheda Samad, MBBS, FCPS (Pediatrics), Professor, Dept. of Pediatrics, Chittagong Medical College, Chittagong.

c. Dr. Jagadish C Das, Professor, Dept. of Neonatology, Chittagong Medical College, Chittagong.

d. Dr. Ferdous Ara, MBBS, Postgraduate student, Dept. of Pediatrics, Chittagong Medical College, Chittagong.

e. Dr. Mohammad Abu Faisal, MBBS, MD (Gastroenterology), Assistant Professor, Dept. of Gastroenterology, Cox's Bazar Medical College, Chittagong.

Address of Correspondence: Dr. Syeda Humaida Hasan, MBBS, DCH, FCPS (Pediatrics), Consultant, Department of Neonatology, Chittagong Medical College Hospital, Chittagong, Bangladesh. E-mail: humaidahasan@yahoo.com, Phone: +088-01816039029

Received: 17 July, 2019

Accepted: 07 November, 2020 dramatically improved by injectable cobalamin. As vitamin B12 deficiency is a treatable condition so it should be ruled out in patient presenting with hyperpigmentation. Early replacement therapy also prevents multisystem involvement like bone marrow failure and demyelinating neurological disease.

Keywords: Hyperpigmentation, Vitamin B12 deficiency

(J Bangladesh Coll Phys Surg 2021; 39: 62-67)

DOI: https://doi.org/10.3329/jbcps.v39i1.50461

skin hyperpigmentation, stomatitis, hair and nail changes. ${ }^{4}$. Early detection and prompt treatment is essential, since it is a reversible cause of bone marrow failure and demyelinating nervous system disease. $^{5}$ Reports of hyperpigmentation as the primary presenting symptom of vitamin B12 deficiency in pediatric age group are sparse in the literature. This is probably the first case report of such manifestation in an adolescent girl from Bangladesh.

\section{Case summery:}

An eleven-year-old adolescent girl was admitted in Department of Paediatrics, Chattogram Medical College Hospital with generalized weakness, weight loss and vomiting for seven months. She had progressive pigmentation started from hands and feet, then became generalized. She was suffering from recurrent oral ulcer and loss of appetite. Local physician noticed pallor and referred her to the tertiary care hospital for better management. Her birth history was uneventful with age appropriate developmental milestone. She was prepubertal (sexual maturity rating stage 1). She used to take mixed family diet. Serving of each food was insufficient for her age and associated vomiting further refrained her from feeding. She frequently 
took egg as protein but meat intake was only once per week. She disliked milk and milk products. She had not taken any drugs which are significantly related with her illness like non steroidal anti-inflammatory drugs(NSAIDs), antimalarials, cytoxic drugs, tetracyclines, heavy metals or proton-pumpinhibitors, histamine-2-receptor blocker for prolonged duration. She belonged to a poor family in rural area. Her father was a carpenter. There was no consanguinity and no significant illness in the family. They lived in a tin shed house and drank water from tube-well. On examination, significant hyperpigmentation in the form of brownish black discoloration was noted which was more marked in hands, feet and around lips. She was pale and hair was sparse, gray with rough texture. Vital signs were stable with no postural hypotension. Examination of the oral cavity showed pigmentation and ulceration present in the inner mucosa. Gum was also pigmented. Tongue was red, smooth with atrophied papillae. Her weight and height was 16 kilogram and 120 centimeter respectively and BMI was $11.1 \mathrm{~kg} / \mathrm{m}^{2}$. Her weight for age was below -2SD, height for age was below -3SD and BMI for age was $-1.97 \mathrm{SD}$ according to CDC growth charts for girls aged 2-20 years. Respiratory, cardiovascular, abdominal, neurological and psyciatric examinations were unremarkable.

She was provisionally diagnosed as a case of Addison Disease. Malnutrition, tuberculosis, arsenicosis were also in our mind as differential diagnoses. There was no history of arsenicosis in the locality. Investigations were done primarily to rule out pallor and hyperpigmentation. Her serum electrolyte and morning cortisol level was within normal ranges which excluded adrenal insufficiency. (Table-1) Adrenocorticotrophic hormone (ACTH) measurement was not available at that time. As literature review suggested that Vitamin B12 deficiency could masquerade Addisonian pigmentation as well as her pallor and glossitis had increased the suspicion of the condition. Hemoglobin was $8.7 \mathrm{gm} / \mathrm{dl}$ and Peripheral Blood Film (PBF) showed findings of anisopiokilocytosis, microcyte and macroovalocyte with some elliptical cell. Her mean corpuscular volume (MCV) was raised (102.20 fl). Tuberculosis screening was negative. Screening for kala-azar was not done as she had no history of travelling in Kala-azar endemic areas and other clinical features were not matched except anemia and dark skin. With a strong suspicion of vitamin B12 deficiency, bone marrow examination and vitamin B 12 assay were done. Bone Marrow examination was emphasized to exclude myelodysplasia and acute leukaemia as both of them may present with macrocytic anemia. There was megaloblastic erythropoiesis in bone marrow and vitamin B-12 level was $85 \mathrm{pg} / \mathrm{ml}$ (Normal level211-911 pg/ml) measured by Automated Chemiluminescence Immunoassay (CLIA). Final diagnosis of vitamin B 12 deficiency was confirmed. Folate level and methylmalonic acid (MMA) could not be measured due to financial problem. Parents did not give consent to do upper GI endoscopy which might give us clue about aetiology of vitamin B 12 deficiency.

She was treated with injectable vitamin B 12 in the form of cyanocobalamin 1000 microgram every other day for 1 week along with vitamin $\mathrm{C}$ and other supplementation for malnutrition. After initiating treatment, improvement was noticed within a week. Her appetite improved, glossitis became better along with gradual fading of pigmentation. Then the patient was discharged with the advice of weekly injection of cyanaocobalamin for a month. Her hematological parameter was improved after 15 days in her $1^{\text {st }}$ follow up. After completion of weekly regimen, oral vitamin B12 supplementation was advised with age appropriate feeding counseling. Oral folate was not given initially to see the response of treatment with injectable vitamin B12 but it was added during discharge of the patient.

Malnutrition was thought to be the primary cause of Vitamin B12 deficiency for her. Other probable causes of vitamin B12 deficiency could not be excluded due to non availability of investigations and financial constrain of the parents. Along with available laboratory finding, rapid improvement after parenteral vitamin B12 strongly supported the diagnosis. 
Table 1: Summary of Patient's Investigations

\begin{tabular}{|c|c|}
\hline Name of the investigations & Patient's value \\
\hline Hemoglobin (gm/dL) & $8.7 \mathrm{~g} / \mathrm{dL}(12-15 \mathrm{~g} / \mathrm{dL})$ \\
\hline Hematocrit(\%) & $30.2 \%(35 \%-45 \%)$ \\
\hline Mean corpuscular volume: MCV (fL) & $102.70 \mathrm{fL}(80-100 \mathrm{fL})$ \\
\hline Reticulocyte count (\%) & $0 \%(0.5-1.5 \%)$ \\
\hline Peripheral blood film & Anisopoikilocytosis, microcyte, macroovalocyte, few myelocyte \\
\hline Serum vitamin B12 (pg/ml) & $85.02 \mathrm{pg} / \mathrm{ml}(211-911 \mathrm{pg} / \mathrm{ml})$ \\
\hline Bone marrow Examination & $\begin{array}{l}\text { Cellularity: normocellular } \\
\text { Myeloid:Erythroid ratio (M:E ratio): normal } \\
\text { Erythropoiesis: Megaloblastic change } \\
\text { Granulopoiesis: Segmented form with giant metamyelocytes } \\
\text { Megakaryopoiesis: Frequent with stipple chromatin }\end{array}$ \\
\hline Serum Electrolyte & 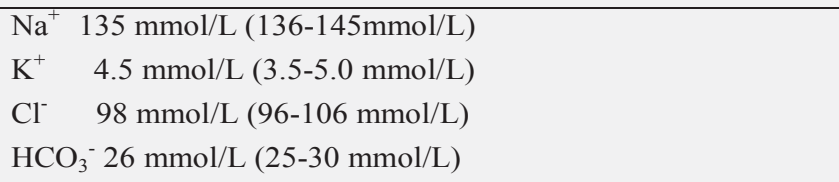 \\
\hline Serum Thyroid Stimulating Hormone ( TSH) & $2.20 \mu \mathrm{IU} / \mathrm{ml}(0.36-5.5 \mu \mathrm{IU} / \mathrm{ml})$ \\
\hline Plasma morning cortisol (microgm/dL) & $17 \mu \mathrm{g} / \mathrm{dL}(5-25 \mu \mathrm{g} / \mathrm{dL})$ \\
\hline Ultrasonogram (USG) of Abdomen & $\begin{array}{l}\text { Normal Study } \\
\text { (Adrenal Gland: not enlarged } \\
\text { Adnexa and Uterus: Normal) }\end{array}$ \\
\hline
\end{tabular}

\section{Illustrations}
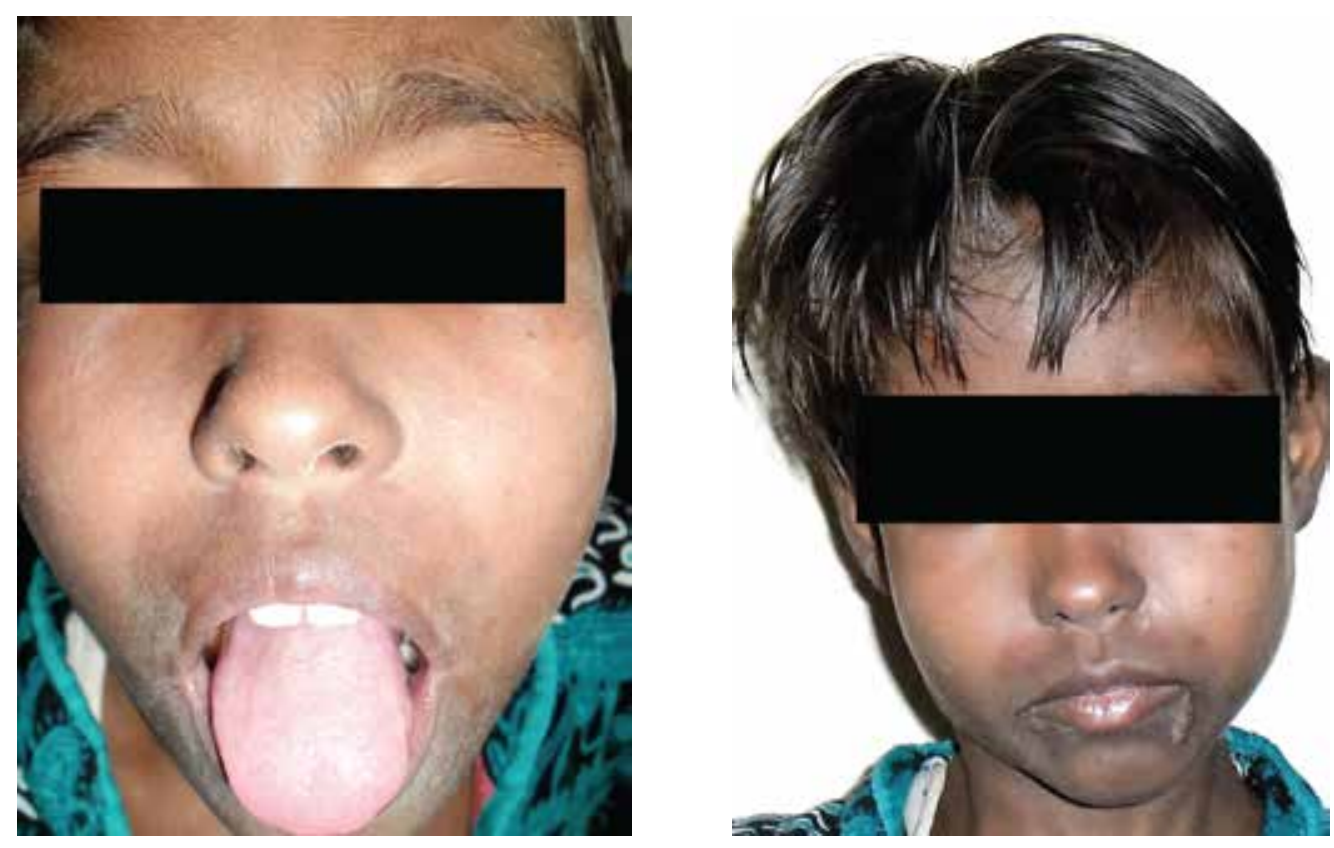

Figure 1: Perioral hyperpigmentation and glossitis

Figure 2: Angular stomatitis along with generalized hyperpigmentation and perioral hyperpigmentation 


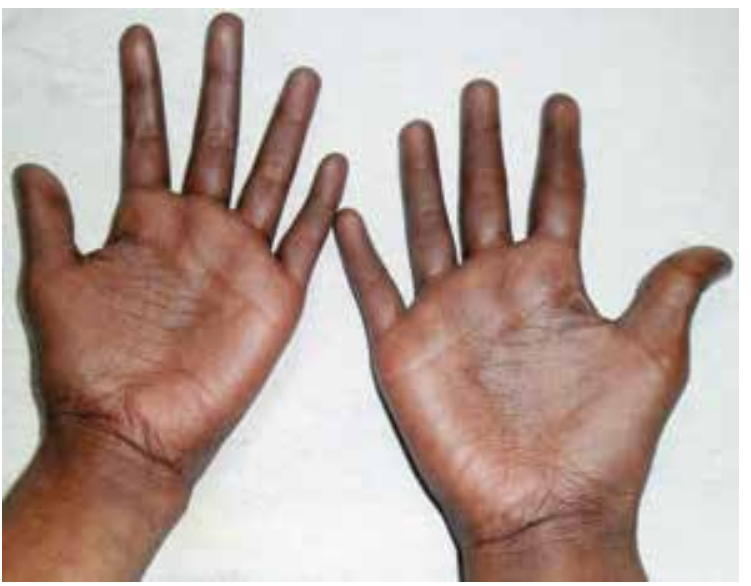

Figure 3: Hyperpigmentation of palm

\section{Discussion:}

Vitamin B12 exists in a number of different chemical forms and is the cofactor for important enzymes. ${ }^{6}$ It is required to convert methyl malonyl CoA to succinyl $\mathrm{CoA}$ and to ensure the activities of methionine synthase which catalyses the conversion of homocysteine to methionine. Vitamin B12 deficiency usually presents with clinical, hematological, neurological and psychiatric manifestations. ${ }^{1}$

Hyperpigmentation may occur due to number of primary skin disorder like melanoma as well as systemic cause like Addison disease, hyperthyroidism, hemochromatosis. Protein energy malnutrition, zinc deficiency and pellagra are known to cause hyperpigmentation. ${ }^{7}$ Cutaneous hyperpigmentation due to B12 deficiency was early described by Baker in $1963 .{ }^{8}$ Later on, few reports were published in Indian subcontinent. Most of them were middle aged female with strict vegetarian diet., ${ }^{7,9}$ Pigmentary changes are reversible and more common in darker-skinned patients. ${ }^{9}$ Extremities are involved especially over the dorsum of the hands and feet, with accentuation over the interphalangeal joint, terminal phalanges (knuckle pad hyperpigmentation), intertriginous areas, oral mucosa and in recent scar. ${ }^{10}$ Some patient also presented with generalized pigmentation. ${ }^{11}$ This girl presented with diffuse hyperpigmentation which was more prominent in extremities, face and around the lips. Pigmentary changes are less in the form of spotty or reticulated in patients of European descent. $^{12}$ The pathophysiology of hyperpigmentation involves i) increased melanin synthesis, and ii) defective melanin transfer from melanocytes to adjacent megaloblastic keratinocytes. Increased tyrosinase activity results in increased melanin synthesis in the presence of decreased reduced glutathione. ${ }^{9}$ There is reversible paradoxical premature greying of hair like our patient. ${ }^{6}$ Other reported muco-cutaneous manifestations are vitiligo, hair changes, glossitis and angular stomatitis. $^{7}$ A review of 89 adult patients who suffered from vitamin B12 deficiency was published where only 5 cases presented with dermatologic manifestation and only 13 cases had glossitis. ${ }^{2}$ The index patient presented with hyperpigmentation with weakness and vomiting as initial symptoms which mimicked Addison disease. Presence of glossitis, grey hair and megaloblastic anemia led to rule out cobalamin deficiency. Some authors suggest to estimate serum B12 level in all the patients with Addison disease as hyperpigmentation and orthostatic hypotension are common symptoms. Both diseases can coexist in the same patient in the context of "polyglandular autoimmune syndromes". 4

Daily Recommended Intake (DRI) of vitamin B12 are 1.8 microgram-2.4 microgram in adolescent age group. ${ }^{13} \mathrm{~A}$ deficiency can be caused by a congenital lack of transcobalamin II, a lack of intrinsic factor, achlorhydria, ileal disease, malnutrition, and malabsorption syndromes. ${ }^{5}$ Breast fed infants of B12 deficient mother are at risk for significant deficiency. ${ }^{1,13}$ Overt tissue deficiency may present during periods of increased requirement for vitamin B12 such as pregnancy and during adolescent growth spurt. ${ }^{14}$ Use of proton pump inhibitor, histamine 2 receptor antagonist and metformin may increase the risk of deficiency though our patient had no such history. ${ }^{13}$ This deficiency has also reported to be associated with other endocrine and autoimmune disorders like pernicious anemia, autoimmune thyroid disease, polycystic overian syndrome (PCOS). ${ }^{4,15}$ We excluded Addison disease, thyroid dysfunction, PCOS in this case by serum electrolyte, basal cortisol, TSH and abdominal USG. Endoscopy was not performed though it was suggested by us as she presented with vomiting. It may find out the aetiology of deficiency. Literature suggest that Helicobacter pylori induced gastritis, gastric and duodenal ulcer may cause B 12 deficiency even in adolescent. ${ }^{5}$ The inadequate dietary intake of vitamin B12 may not be apparent until a detailed dietary assessment is performed. ${ }^{14}$ The girl was malnourished. Though she took mixed diet, it was difficult to measure the nutritional value of that diet. 
It is well known that cobalamin deficiency happens in vegetarian but studies conducted in India revealed more than $75 \%$ affected patients were non-vegetarian. ${ }^{3,16}$ It suggests that it is difficult to rule out B12 deficiency even in non-vegeterian population in developing countries.

There is no gold standard test to diagnose cobalamin deficiency. ${ }^{17}$ The increase of MCV is the earliest manifestation of megaloblastosis. Red blood cells appear larger than normal, and some of them may lose the central pale area; furthermore, there may be anisocytosis and poikilocytosis. Macroovalocytes with incompletely extruded nuclei sometimes circulate in the peripheral blood. ${ }^{18}$ These findings were similar with our patient. In the bone marrow, there may be reduced M:E ratio from 3:1 to $1: 1 .^{18}$ The girl had normal $\mathrm{M}: \mathrm{E}$ ratio with megaloblastic erythropoiesis and giant metamyelocyte. Hypersegmented polymorphonuclear neutrophils (PMN), Howell-Jolly bodies and Cabot rings in RBC were not found. MMA elevation is more sensitive in non anemic B 12 deficency than homocysteine level elevation as the later is influenced by low vitamin B 6 and low folate level. ${ }^{19}$ Though elevated MMA or homocysteine are not conclusive proof of cobalamin deficiency. ${ }^{17}$ Traditionally, deficiency was defined as a plasma concentration of vitamin B $12<200 \mathrm{pg} / \mathrm{ml}$ (148 pmol/L). ${ }^{9}$ This cut point of vitamin B12 level has $95-97 \%$ sensitivity to diagnose deficiency. ${ }^{17}$ This patient had only $85.02 \mathrm{pg} / \mathrm{ml}$ of cobalamin. It is matched with a previous study where mean B12 level was $86+8.5 \mathrm{pg} / \mathrm{ml}$ in patients presented with pigmentation. ${ }^{16}$ Measurement of homocysteine, MMA and Intrinsic factor antibody were not done due to logistic constraints. Another limitation of the study was non estimation of folate level. Folate deficiency was less frequently reported with pigmentary change and the cost was also unbearable for the parents. Moreover, it is recommended to do red blood cell folate levels instead of serum folate levels which tend to be increased with B 12 deficiency due to impairment of methionine synthase pathway. ${ }^{6,19}$ Red blood cell folate level was also not available in our center.

Usually, $1 \mathrm{mg} /$ day of cobalamin given intramuscularly for a week, followed by $1 \mathrm{mg} /$ week for four weeks and then $1 \mathrm{mg} / \mathrm{month}$ for life, is an appropriate therapeutic regimen. Obviously, therapy should be started after the vitamin deficiency has been established and the causes thoroughly looked for. Oral daily doses of 1-2 $\mathrm{mg}$ seem also to be suitable for patients refusing parenteral therapy. ${ }^{18}$ Injection or high oral doses are preferred as absorption often cannot be determined. ${ }^{17}$ For better absorption and better compliance, we chose parenteral therapy for our hospitalized patient. The side effect of injectable cyanocobalamin is also minimal. ${ }^{17}$ She had better appetite, reduced pigmentation and glossitis after first week of treatment. We did not give empiric folic acid before confirmation of diagnosis. In addition to understand the treatment response by cobalamin, it was also in our mind that folate level may be high in the blood in B12 deficiency. High serum folate levels during vitamin B12 deficiency exacerbate anemia and worsen cognitive symptoms..$^{20}$ After $1^{\text {st }}$ week of daily B12 injection, she was discharged with weekly injection along with folic acid. Her hematological profile also improved which supports previous data. ${ }^{5,11,18}$

\section{Conclusion:}

The triad of hyperpigmentation, anemia and glossitis led to the diagnosis of vitamin B12 deficiency in this case. Pigmentation as initial presentation is not so common in vitamin B12 deficiency even though it is important for physicians to have a high index of suspicion to rule out vitamin B 12 deficiency even if in non-vegeterian population. Early replacement therapy may reverse the symptom and help to prevent development of neurological complications.

\section{References:}

1. Guez S, Chiarelli G, Menni F, Salera S, Principi N, Esposito S. Severe vitamin B12 deficiency in an exclusively breastfed 5-month-old Italian infant born to a mother receiving multivitamin supplementation during pregnancy. BMC Pediatrics 2012; 12: 85. [doi:10.1186/1471-2431-12-85]

2. Rusher DR, Pawlak R. A Review of 89 Published Case Studies of Vitamin B12 Deficiency. J Hum Nutr Food Sci 2013; 1(2): 1008.

3. Sen K, Sinhamahapatra P, Lalhmachhuana J, Ray S. A study of clinical profile of vitamin B12 deficiency with special reference to dermatologic manifestations in a Tertiary Care Hospital in sub-Himalayan Bengal. Indian J Dermatol 2015; 60:419. [doi:10.4103/0019-5154.160506]

4. Cherqaoui R, Husain M, Madduri S, Okolie P, Nunlee-Bland G, Williams J. A Reversible Cause of Skin Hyperpigmentation and Postural Hypotension. Case Rep Hematol 2013; 2013: 680459, [doi: $10.1155 / 2013 / 680459]$ 
5. Anitha P, Sasitharan R, Thambarasi T, Krithika P, Mohan M, Venkataraman P et al. Vitamin B12 deficiency presenting as pancytopenia and retinopathy in a young boy-Helicobacter pylori, a novel causative agent. AMJ 2014; 7:3: 143-148. [doi:10.4066/AMJ.2014.1872]

6. Jithendriya M, Kumaran S, Bhat I. Addisonian Pigmentation and Vitamin B Deficiency: A Case Series and Review of the Literature. Cutis 2013; 92: 94-99.

7. Santra G, Paul R, Ghosh SR, Chakraborty D, Das S, Pradhan $\mathrm{S}$ et al. Generalised Hyperpigmentation in Vitamin B12 Deficiency. J Assoc Physicians India 2014; 62: 714-716.

8. Baker SJ, Ignatius $\mathrm{M}$, Johnson $\mathrm{S}$, Vaish SK. Hyperpigmentation of skin. A sign of vitamin-B12 deficiency. Br Med J. 1963; 1: 1713-1715.

9. Agrawala RK, Sahoo SK, Choudhury AK, Mohanty BK, Baliarsinha AK. Pigmentation in vitamin B12 deficiency masquerading Addison's pigmentation: A rare presentation. Indian J Endocr Metab 2013; 17: S254-6. [doi: 10.4103/2230-8210.119591]

10. Chakrabarti S. A Curious Case of Fever and Hyperpigmentation. J Clin Diagn Res 2015; 9: OD01-OD03. [doi: 10.7860/JCDR/2015/11027.5379]

11. Hoffman CF, Palmer DM, Papadopoulos D.Vitamin B12 Deficiency: A Case Report of Ongoing Cutaneous Hyperpigmentation. Cutis 2003; 71:127-130.

12. Aroni K, Anagnostopoulou K, Tsagroni E, Ioannidis E .Skin Hyperpigmentation and Increased Angiogenesis Secondary to Vitamin B12 Deficiency in a Young Vegetarian Woman. Acta Derm Venereol 2008; 88: 191-192. [doi: 10.2340/00015555-0377]

13. Sachdev HPS, Shah D. Vitamin B Complex Deficiencies and Excess. In. Kliegman RM, Stanton BF, Geme JW, Schor NF, Behrman RE eds. Nelson textbook of Pediatrics. 20 $0^{\text {th }}$ edition. Philadelphia: Elsevier Saunders 2016; 321-328.

14. O'Gorman P, Holmes D, Ramanan AV, Bose-Haider B, Lewis MJ, Will A. Dietary vitamin B12 deficiency in an adolescent white Boy. J Clin Pathol 2002;55: 475-476.

15. Gourgari E, Nella A A, Lodish M, Stratakis CA, Yanovski JA. Case Report: Vitamin B12 deficiency in an adolescent female with Polycystic Ovarian Syndrome. Eur J Obstet Gynecol Reprod Biol. 2014; 179: 254. [doi:10.1016/j.ejogrb.2014.04.036]

16. Arora R, Dalal A. Cutaneous Manifestations of Vitamin B12 Deficiency. IJIRSET 2016;5 :3: 3780-3786.

17. Carmel R. How I treat cobalamin (vitaminB12) deficiency. BLOOD 2008; 112: 2214-2221. [doi:10.1182/blood-2008-03-040253]

18. Briani C, Torre CD, Citton V, Manara R, Pompanin S, Binotto $G$ et al. Review Cobalamin Deficiency: Clinical Picture and Radiological Findings. Nutrients 2013; 5: 4521-4539. [doi:10.3390/nu5114521]

19. Aslinia F, Mazza JJ, Yale SH. Megaloblastic anemia and other causes of macrocytosis. Clin Med Res 2006; 4(3):236-241. [doi:10.3121/cmr.4.3.236]

20. Johnson MA. If high folic acid aggravates vitamin B12 deficiency what should be done about it? Nutr Rev 2007;65: 451-8. [PubMed ID 17972439] 